\title{
Differential Contributions of the Left and Right Inferior Parietal Lobules to Number Processing
}

\author{
F. Chochon \\ INSERM U334, Orsay, France
}

L. Cohen

INSERM U334, Orsay and Hôpital de la Salpêtrière, Paris, France

\author{
P. F. van de Moortele and S. Dehaene \\ INSERM U334, Orsay, France
}

\begin{abstract}
We measured cerebral activation with functional magnetic resonance imaging at 3 Tesla while eight healthy volunteers performed various number processing tasks known to be dissociable in brain-lesioned patients: naming, comparing, multiplying, or subtracting single digits. The results revealed the activation of a circuit comprising bilateral intraparietal, prefrontal, and anterior cingulate components. The extension and
\end{abstract}

lateralization of this circuit was modulated by task demands. The intraparietal and prefrontal activation was more important in the right hemisphere during the comparison task and in the left hemisphere during the multiplication task and was intensely bilateral during the subtraction task. Thus, partially distinct cerebral circuits with the dorsal parietal pathway underlie distinct arithmetic operations.

\section{INTRODUCTION}

Previous neuropsychological and imaging work has emphasized the crucial role of the left inferior parietal lobule in number processing. In brain-lesioned cases, this area is the main lesion site causing acalculia, a selective deficit in arithmetic (Dehaene \& Cohen, 1997; Gerstmann, 1940; Takayama, Sugishita, Akiguchi, \& Kimura, 1994). Furthermore, this region is activated when normal subjects perform simple mental calculations such as single-digit multiplication (Dehaene et al., 1996), approximation (Dehaene, Spelke, Stanescu, Pinel, \& Tsivkin, 1999), or serial subtraction (Roland \& Friberg, 1985; Rueckert et al., 1996). However, those brain-imaging studies have also repeatedly evidenced a concomitant activation of the homologous inferior parietal region of the rigbt hemisphere in the same tasks.

The present brain-imaging study was designed to throw some light on the respective contributions of the left and right inferior parietal areas to number processing. We postulated that different number-processing tasks are associated with distinct cerebral circuits and, in particular, with distinct patterns of lateralization within the parietal lobe. We selected tasks based on two criteria: their frequent dissociation in single-case studies of pa-

tients with number-processing deficits and the predictions that could be derived about them from current theories of number processing.

\section{Dissociations between Operations in Brain-Lesioned Patients}

The main motivation for our study lies in the finding that, in many single-case studies of patients with numberprocessing deficits, different arithmetic operations such as naming, comparing, multiplying, and subtracting digits are not equally impaired. First, digit naming is often selectively preserved in "Gerstmann syndrome" patients with acalculia stemming from a left inferior parietal lesion and who experience severe difficulties in mental arithmetic. Such patients may fail to compute, for instance, 31 or $9 \times 8$, yet experience no difficulty in reading the numbers aloud (Dehaene \& Cohen, 1997; Takayama et al., 1994). Conversely, digit naming can be impaired in patients who otherwise have no difficulty in mental arithmetic (Cipolotti \& Butterworth, 1995), including patients with pure alexia (Cohen \& Dehaene, 1995). This double dissociation has led to the suggestion that naming a digit can occur without necessarily access- 
ing its semantic code (Cipolotti \& Butterworth, 1995; Dehaene \& Cohen, 1995).

Dissociations between arithmetic operations are also on record. The most frequent dissociation is between comparison and calculation. Severely aphasic and acalculic patients with major left-hemispheric lesions, even if they cannot name, add, subtract, or multiply digits, may still decide which of two numbers is larger (Dehaene \& Cohen, 1991; Grafman, Kampen, Rosenberg, Salazar, \& Boller, 1989). The hypothesis that number comparison is supported at least in part by right-hemispheric processes receives support from studies of split-brain patients. When pairs of digits are flashed in the left visual field, therefore contacting the right hemisphere only, splitbrain patients are generally unable to read them aloud or calculate with them. Yet they can decide whether the two digits are identical or which of them is larger (Cohen \& Dehaene, 1996; Gazzaniga \& Hillyard, 1971; Gazzaniga \& Smylie, 1984; Seymour, Reuter-Lorenz, \& Gazzaniga, 1994). It is noteworthy that, when digits are flashed in their right hemifield and hence to their left hemisphere, these patients can readily name, compare, or calculate with them. Hence, the ability to compare two numbers seems to be redundantly available to both hemispheres, whereas naming and calculating abilities seem to require a left-hemispheric contribution.

Even two seemingly similar arithmetic operations, such as subtraction and multiplication, can be dissociated. There are several cases on record that have a selective deficit of addition and multiplication in the face of relatively spared subtraction (Dagenbach \& McCloskey, 1992; Lampl, Eshel, Gilad, \& Sarova-Pinhas, 1994; McNeil \& Warrington, 1994; Pesenti, Seron, \& van der Linden, 1994 ) or the converse (Delazer \& Benke, 1997). Recently, Dehaene and Cohen (1997) have reported a double dissociation between parietal and subcortical acalculia. A patient with a left subcortical lesion suffered from a serious impairment of rote memory for multiplication problems, whereas her performance in solving addition and subtraction problems was much better. Conversely, another patient with an inferior parietal lesion and Gerstmann's syndrome failed to solve very simple addition and subtraction problems, whereas he performed significantly better when retrieving rote multiplication facts. ${ }^{1}$

Although such cases clearly suggest that distinct arithmetic operations rely partly on dissociable brain circuits, they provide little information about their anatomical localization. Most of these studies were framed in a cognitive neuropsychological perspective and did not specifically look for the anatomical substrates of the deficits. Furthermore, brain lesions are often too large to allow for precise anatomical inferences, and there are still too few cases in the literature to warrant a statistical meta-analysis of lesion localization. At present, the only solid anatomical conclusions that may be drawn from the neuropsychological litterature are that (1) the left inferior parietal region is critical for most calculation deficits, particularly subtraction, although number naming, comparison, and even multiplication may be relatively preserved and (2) the right parietal area is generally not associated with specific number-processing deficits in clinical neuropsychological practice, although impairments of number comparison (Rosselli \& Ardila, 1989) and of the comprehension of arithmetic relations (Langdon \& Warrington, 1997) have been reported in some group studies of patients with right parietal lesions.

\section{A Model of Number Processing Circuits}

A model of the functional and anatomical architecture of the number processing system, the triple-code model (Dehaene, 1992; Dehaene \& Cohen, 1995), can partially explain the occurrence of dissociations between operations. The model assumes that numbers can be represented in the human brain in three distinct formats: as Arabic numerals, as sequences of words, and as analogical representations of the corresponding numerical quantity (Figure 1). In the visual Arabic code, numbers are encoded as strings of digits on an internal visuospatial scratchpad. This representation, putatively involving the left and right ventromesial occipito-temporal pathways, allows for the identification of Arabic numerals and subserves multidigit operations and parity judgments. In the verbal code, numbers are encoded as syntactically

Figure 1. Schematic diagram of the architecture of the triple-code model.

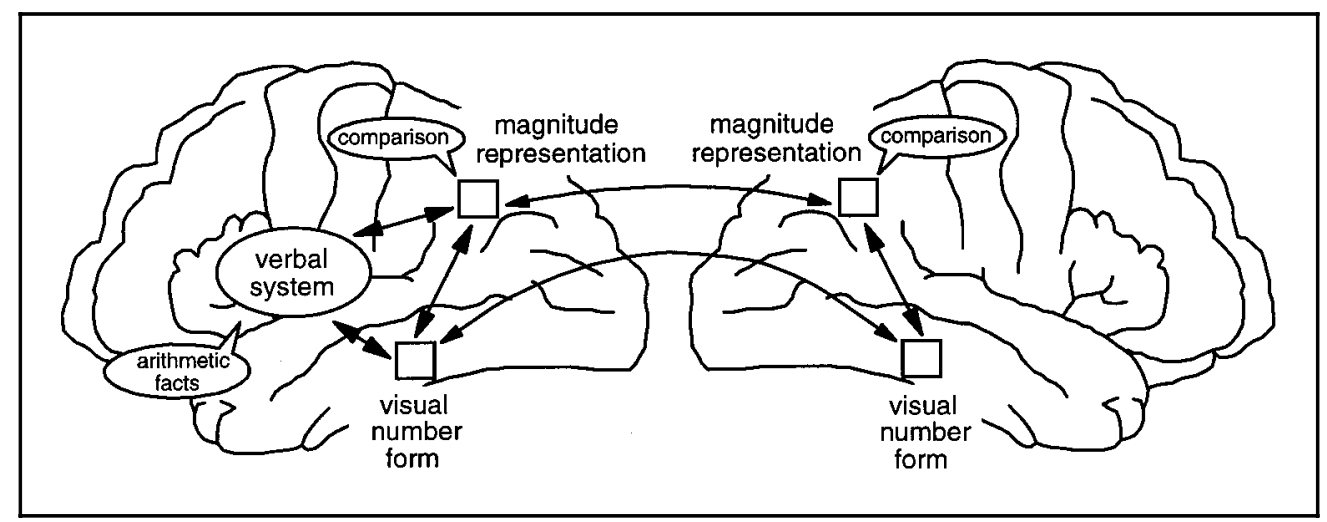


organized sequences of words (e.g., twenty-four). This representation, supported by left perisylvian language areas, allows for the comprehension and production of spoken numerals. It is postulated to be the obligatory entry code for accessing stored tables of rote arithmetic facts, encoded in the form of short sentences in verbal memory (e.g., two times three, six). Finally, in the magnitude code, numbers are represented as analogical quantities on an oriented line. Numerical relations, such as knowing that 9 is larger than 5, are then implicitly represented by proximity relations on the number line. Thus, this semantic code, putatively involving the left and right inferior parietal lobules, supports number comparison and other semantic manipulations of numerical quantities.

The model assumes that these three cardinal representations are linked by direct transcoding routes that allow numbers to be rapidly translated internally to and from the different formats (see Figure 1). According to this model, two main routes are therefore available to solve single-digit arithmetical problems presented in Arabic format. First, there is a direct route, in which the input numerals are converted into a verbal format and then a rote verbal memory store is accessed for arithmetic facts. This route is typically used for overlearned facts such as single-digit addition and multiplication problems, for which a stored "table" is available. The second route is an indirect semantic route, in which mental manipulations of numerical quantities are used to compute the results. This pathway is used whenever rote verbal knowledge of the answer is lacking, most typically for subtraction problems. According to the model, quantity processing relies on the inferior parietal cortex, in connection with the left perisylvian language network whenever verbal output is required.

\section{Experimental Design}

Based on the neuropsychological literature and the triple-code model, digit naming, comparison, multiplication, and subtraction were selected as contrastive tasks for our brain-imaging experiments. In all four tasks, single digits between 1 and 9 were presented visually at a rate of one every 2 sec. In the naming task, subjects named the target digits. In the comparison task, they had to compare the target digits to 5, responding with the words "larger" or "smaller." In the multiplication task, subjects multiplied the target digits by 3 . Finally, in the subtraction task, they subtracted the target digits from 11.

The control task was a simple letter-naming task: A single letter from A to I was flashed with the same timing as the digit stimuli, and subjects simply responded with the letter's name. This task controlled for the visual and response requirements of the arithmetic tasks. Contrasting the functional magnetic resonance imaging (fMRI) responses during arithmetic relative to control should isolate the cerebral networks involved in the internal transformation of numerical information.

To avoid head movement in the fMRI scanner, the subjects were asked to utter the responses subvocally "in their head," which prevented us from measuring their performance. To provide reference behavioral data about the tasks, eight additional subjects were asked to perform the same tasks with overt responses outside the scanner, while their response times were recorded with a voice key.

\section{Predictions for Brain Activity during Arithmetic}

Three critical predictions of the triple-code model were examined in the fMRI results:

1. Digit naming should involve a direct, asemantic transcoding route from the visual number form to the left-hemispheric verbal system, without requiring access to the quantity system. Hence, little or no activation should be found in left and right parietal cortices during digit naming.

2. Number comparison should activate a bilateral inferior parietal network. The evidence for preserved number comparison in the right hemisphere of split-brain patients and in cases of large left-hemispheric lesions leads us to predict a strong activation of the right inferior parietal area during number comparison, above and beyond any left-hemispheric activation.

3. Multiplication and subtraction should show partially different activation patterns. Subtraction, which is not generally learned by rote verbal strategies and can be selectively impaired following inferior parietal lesions, should yield greater activation of the quantity system than multiplication. Conversely, multiplication, which is generally stored in rote verbal memory, should involve left-hemispheric language areas and should be much more strongly lateralized to the left hemisphere than subtraction.

\section{RESULTS}

\section{Behavioral Findings}

Subjects tested outside the fMRI scanner made 0.2 and $0.7 \%$ errors in the letter and digit naming tasks, respectively; $0.9 \%$ errors in the comparison task; and $2 \%$ errors in the multiplication and subtraction tasks. Only 10 correct trials out of 2240 trials yielded response latencies longer than 2 sec (all in the 2- to 3 -sec range; 3 trials in multiplication and 7 in subtraction). Thus, all five experimental tasks were performed with high accuracy within the time limits imposed by the fMRI procedure (one stimulus every $2 \mathrm{sec}$ ).

Mean naming latencies were computed on the basis of correct trials, excluding five additional trials on which the response failed to trigger the voice key. Letter- and digit-naming latencies did not differ (mean $=455 \mathrm{msec}$ 
and 451 msec, respectively; $F(1,7)<1)$. Both naming tasks were faster than digit comparison (mean $=\mathbf{5 4 0}$ msec; $\left.F_{\mathbf{S}}(1,7)>23 ; P_{\mathrm{s}}<0.002\right)$. Comparison was faster than multiplication $($ mean $=807 \mathrm{msec} ; F(1,7)=15.5$; $P=0.0056)$, which itself was faster than subtraction $($ mean $=919$ msec $; F(1,7)=6.73 ; P=0.036)$.

\section{Brain-Imaging Findings}

We first determined which areas are involved in all four number processing tasks relative to the control task of letter naming. Activation peaks, together with their $Z$ score, their Talairach coordinates, and the corresponding Brodmann's areas (BAs) are listed in the Tables 1 and 2. A distributed bilateral parietal, frontal, and anterior cingulate network was activated. In the parietal lobe, activation was concentrated along the banks of the intraparietal sulcus, extending inferiorily into the superior part of the inferior parietal lobule (BA 39/40) and anteriorily in the depth of the postcentral sulcus. The other active areas were the anterior cingulate gyrus (BA 32 ), the bilateral frontal lobes, including the inferior gyrus (BA 44/45), the middle dorsolateral gyrus (BA 9/46), and the right superior gyrus (BA 6/8), as well as the left precentral gyrus (BA 6) and the mesial frontal gyrus (supplementary motor area, or SMA, and BA 8/11).

Figure 2 demonstrates the range of interindividual variation for this contrast (number processing versus letter naming). Five subjects showed a clearly bilateral pattern of activation in the inferior parietal lobe, whereas three subjects showed significant activation only in the left intraparietal region. Nevertheless, in all cases, the activation during number processing followed the banks of the middle sectors of the intraparietal sulcus, often extending anteriorily into the depth of the postcentral sulcus, particularly in the right hemisphere.

We then analyzed separately the four contrasts defined by each numerical task versus control (see Figure 3 and Tables 1 and 2). During digit naming versus control, only the right inferior frontal gyrus and the right mesial frontal gyrus were weakly activated. During digit comparison versus control, a parieto-fronto-cingular network was again detected. The activated areas were, in the right parietal lobe, the postcentral gyrus and/or sulcus, and the intraparietal sulcus. In the left parietal lobe, the intraparietal sulcus and the superior part of the inferior parietal lobule were activated. The activated frontal areas consisted of the left inferior frontal gyrus, the left mesial frontal gyrus, the left precentral gyrus, the right inferior frontal gyrus, and the right precentral gyrus. The anterior cingulate gyrus and the right putamen were also activated.

During multiplication versus control, a network similar to the one observed during number comparison was activated. However, although activation remained bilateral, there was now a clear predominance in the left hemisphere (see Tables 1 and 2). The active parietal areas were both intraparietal sulci, the right postcentral gyrus and/or sulcus, and the superior part of the left inferior parietal lobule. The anterior cingulate gyrus was also

Table 1. Coordinates and Z Scores of Significant Activation Peaks in the Parietal Lobe

\begin{tabular}{|c|c|c|c|c|c|c|}
\hline Brain Area & $\begin{array}{c}\text { Coordinates } \\
\text { in Talairach } \\
\text { Space }\end{array}$ & $\begin{array}{l}\text { All Tasks } \\
\text { vs. Control }\end{array}$ & $\begin{array}{l}\text { Digit Naming } \\
\text { vs. Control }\end{array}$ & $\begin{array}{c}\text { Comparison } \\
\text { vs. Control }\end{array}$ & $\begin{array}{c}\text { Multiplication } \\
\text { vs. Control }\end{array}$ & $\begin{array}{r}\text { Subtraction } \\
\text { vs. Control }\end{array}$ \\
\hline $\begin{array}{l}\text { R postcentral sulcus/ } \\
\text { anterior intraparie- } \\
\text { tal sulcus }\end{array}$ & $42, \quad 30,45$ & $\begin{array}{l}8.21 \\
(4 \mathrm{~S})\end{array}$ & & $\begin{array}{l}6.38 \\
(3 \mathrm{~S})\end{array}$ & $\begin{array}{l}6.01 \\
(3 \mathrm{~S})\end{array}$ & $\begin{array}{l}7.97 \\
(3 \mathrm{~S})\end{array}$ \\
\hline $\begin{array}{l}\mathrm{R} \text { intraparietal sulcus } \\
\text { (middle part) }\end{array}$ & $42, \quad 39,39$ & $\begin{array}{l}7.64 \\
(4 \mathrm{~S})\end{array}$ & & & & $\begin{array}{l}7.37 \\
(5 \mathrm{~S})\end{array}$ \\
\hline $\begin{array}{l}\mathrm{R} \text { intraparietal sulcus } \\
\text { (middle part) }\end{array}$ & $39, \quad 42,42$ & $\begin{array}{l}7.70 \\
(5 \mathrm{~s})\end{array}$ & & $\begin{array}{l}4.89 \\
(3 \mathrm{~S})\end{array}$ & $\begin{array}{l}5.75 \\
(2 \mathrm{~S})\end{array}$ & $\begin{array}{l}7.64 \\
(6 \mathrm{~s})\end{array}$ \\
\hline $\begin{array}{l}\mathrm{L} \text { intraparietal sulcus } \\
\text { (posterior part) }\end{array}$ & $39, \quad 54,48$ & $\begin{array}{l}7.95 \\
(8 \mathrm{~S})\end{array}$ & & $\begin{array}{l}5.35 \\
(5 \mathrm{~S})\end{array}$ & $\begin{array}{l}7.53 \\
(5 \mathrm{~S})\end{array}$ & $\begin{array}{l}7.83 \\
(7 \mathrm{~S})\end{array}$ \\
\hline $\begin{array}{l}\mathrm{L} \text { intraparietal sulcus } \\
\text { (posterior part) }\end{array}$ & $27,66,42$ & $\begin{array}{l}8.18 \\
(7 \mathrm{~S})\end{array}$ & & $\begin{array}{l}4.69 \\
(25)\end{array}$ & $\begin{array}{l}7.17 \\
(4 S)\end{array}$ & $\begin{array}{l}7.93 \\
(7 \mathrm{~S})\end{array}$ \\
\hline
\end{tabular}

Note: The number of subjects showing a significant activation in this anatomical area appears in parentheses. 
Table 2. Coordinates and Z Scores of Significant Activation Peaks Outside the Parietal Lobe

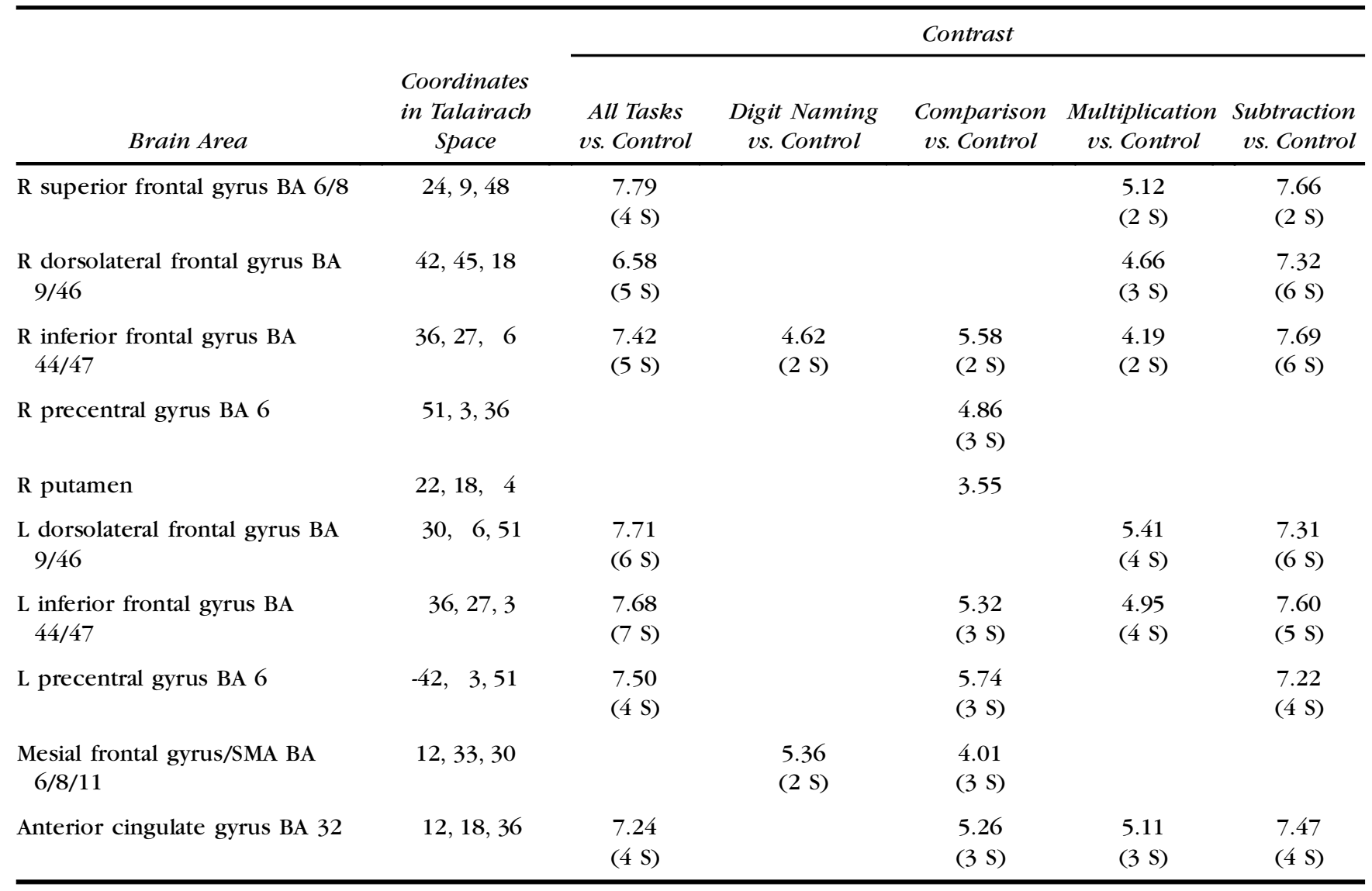

Note: The number of subjects showing a significant activation in this anatomical area appears in parentheses. Anatomical labels should be interpreted cautiously because they were obtained by reporting the group activation peaks on the Talairach atlas. BA is the approximate Brodmann's area.

activated. In the frontal lobe, both inferior frontal gyri were activated, together with both dorsolateral frontal gyri and the right superior frontal gyrus. The individual analysis also detected activation in the mesial frontal cortex in five subjects, although this localization did not appear in the group analysis.

In subtraction versus control, the same parieto-frontocingular network was now greatly activated, equally in both hemispheres. Areas of activation encompassed both intraparietal sulci, the superior part of both inferior parietal gyri, and the right postcentral sulcus. The left postcentral sulcus was also activated in five subjects, although not in the group analysis. In the frontal lobe, both inferior frontal gyri were activated together with the dorsolateral frontal gyri, the left precentral gyrus, and the right superior frontal gyrus. The mesial frontal gyri were also activated in five subjects, as in the multiplication task. Finally, the anterior cingulate gyrus also showed activation. To determine which of these activation patterns were significantly different across tasks, we then directly contrasted the numerical tasks with one another (see Methods). Although all 12 pairs of such comparisons were analyzed, the results turned out to be relatively simple because the occurrence of additional activation followed a strictly hierarchical pattern. The four numerical tasks could be placed in the order Naming < Comparison < Multiplication < Subtraction. There never was a significant activation in any brain region when a given task was contrasted with a task higher in the hierarchy. We therefore only report the six comparisons in which a significant difference was found (see Figure 4 and Table 3 ).

During comparison versus digit naming, activation was detected only in the right postcentral sulcus. At the next level in the hierarchy, for multiplication versus digit naming, activation largely predominated in the left hemisphere, in the left precentral gyrus and sulcus, and along all of the left intraparietal sulcus. The only right-hemispheric activation was in the postcentral sulcus. When multiplication was contrasted to comparison, however, only the left intraparietal activation remained significant. Finally for subtraction versus digit naming, the same fronto-cingulo-parietal network described in subtraction versus letter naming was activated in both hemispheres, although with a lower intensity. When subtraction was contrasted with comparison, the same network was again bilaterally activated, with the sole exception of the absence of activation in the right postcentral sulcus. 
Figure 2. Individual analysis of the eight subjects during all four number processing tasks versus control, at $p=$ 0.001 , corrected at 0.1 . The in dividual anatomical images of all the subjects have been normalized. For each image, the subject's sex ( $m$ or $\mathrm{f}$ ) and age as well as the axial coordinate of the slice $(z)$ are provided.

Figure 3. Group analysis of the comparison, multiplication, and subtraction tasks versus their control, at $p=0.001$, corrected at 0.05 . $Z$ gives the Talairach coordinate of the slices. $\mathrm{m}, 20$ ys

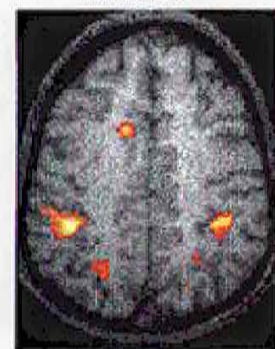

$\mathrm{z}=39$

f, 20 ys

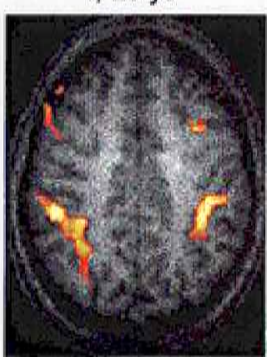

$z=45$ $m, 23$ ys

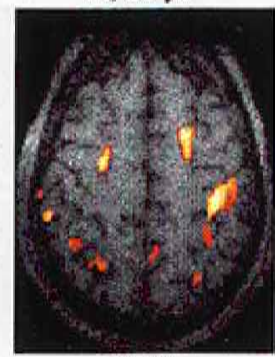

$z=45$

f. 21 ys

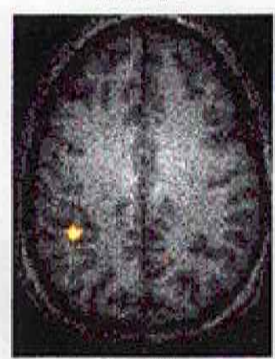

$z=33$ $m, 22$ ys

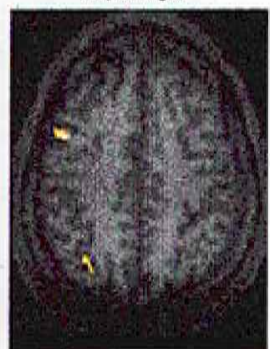

$z=51$

$t_{1} 29$ ys

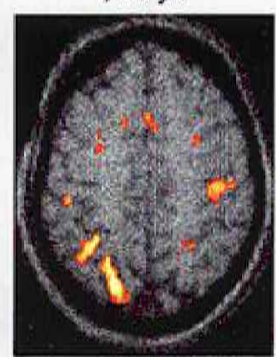

$z=45$ $\mathrm{m}, 20 \mathrm{ys}$

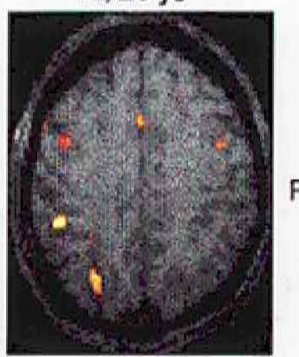

$z=39$

f, 23 ys

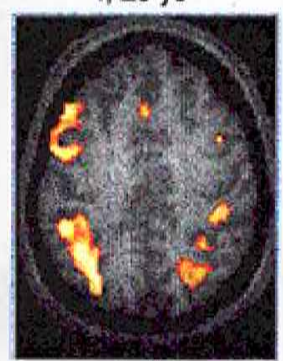

$z=45$
L

\section{Comparison vs control}
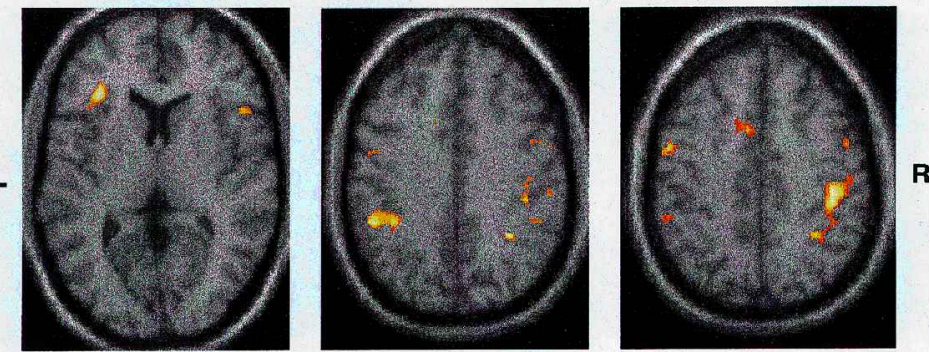

Multiplication vs control
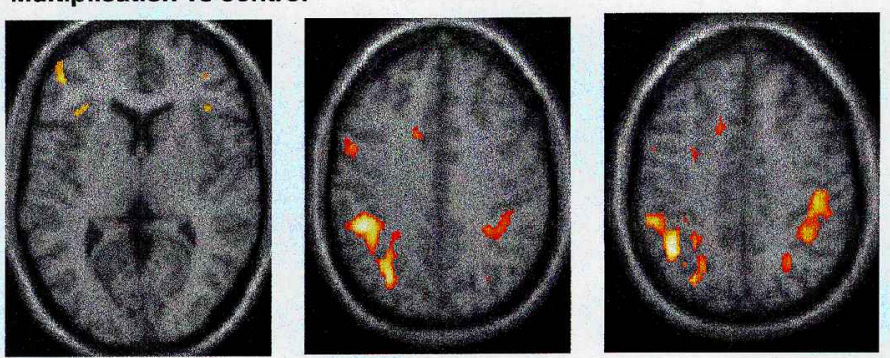

Subtraction vs control
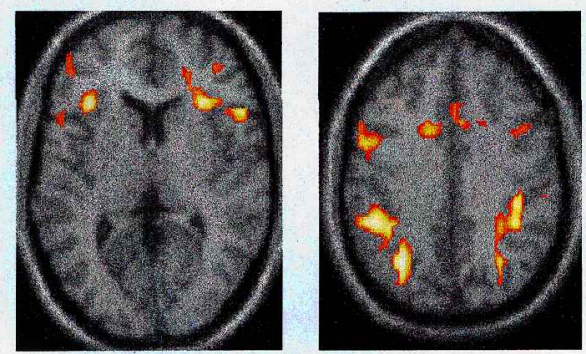

$z=39$

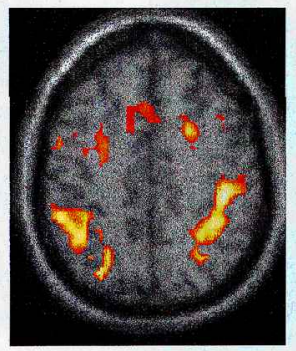

$z=45$ 
Figure 4. Comparisons across the four numerical tasks. The glass-brain views showed the active areas for contrasts comparing any two numerical tasks $(p<0.001$, corrected $p<0.05)$. Contrasts were masked by the corresponding contrast of the top task relative to the letter-naming control $(p<0.001)$ to focus only on activations and cancel out deactivations relative to control. Six contrasts showed significant effects, whereas the six contrasts in the opposite direction showed no significant difference.

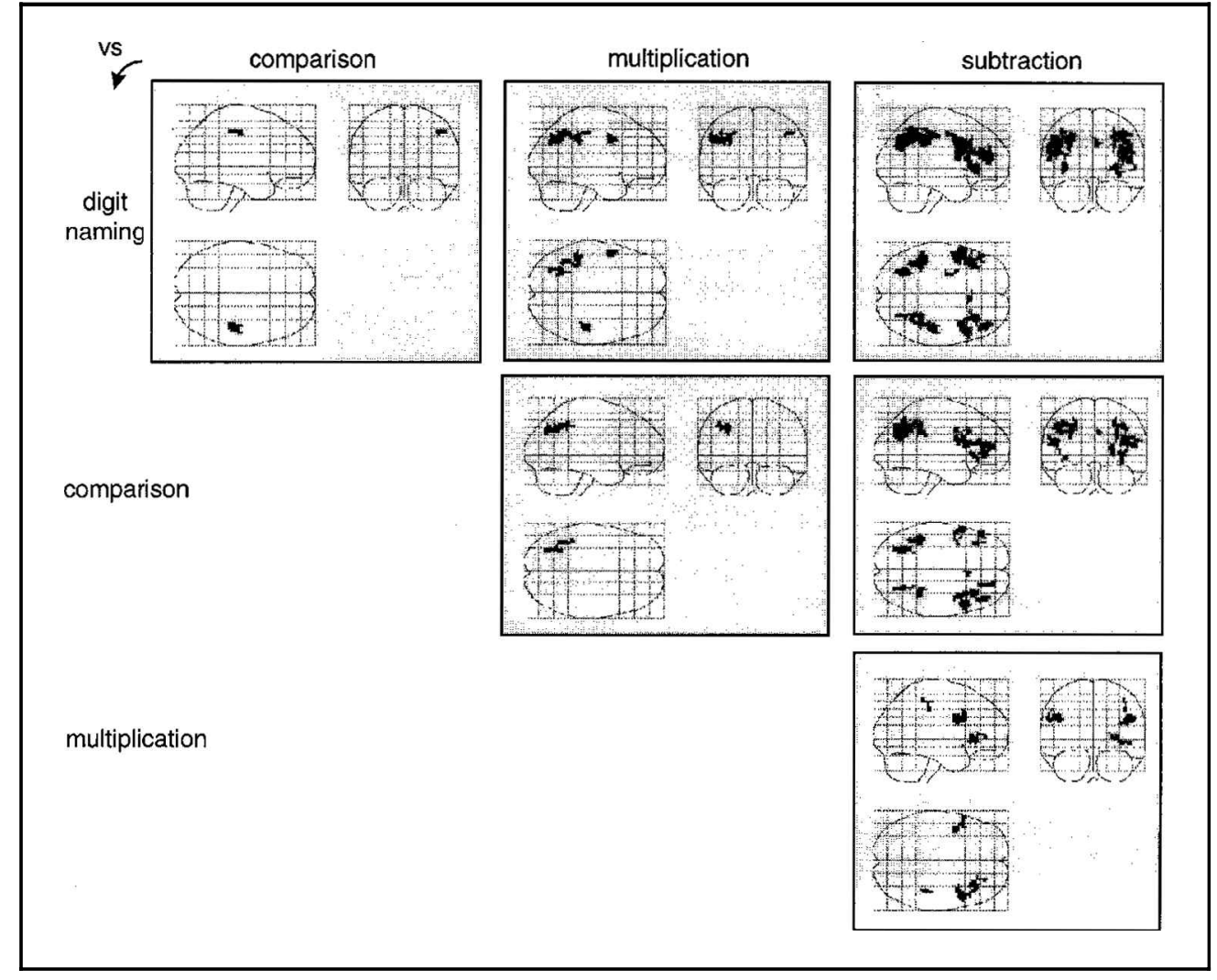

When subtraction was contrasted with multiplication, conversely, the parietal activation was now restricted to the right hemisphere, in the right anterior intraparietal/postcentral region. In the frontal lobe, both inferior gyri were activated, together with the right dorsolateral gyrus.

\section{DISCUSSION}

We begin by briefly summarizing the results. A distributed network of brain regions including parietal, frontal, and anterior cingulate areas was engaged during number processing. However, there were important differences as a function of task demands. First, the parieto-frontocingular network was only activated when subjects were engaged in active number manipulations tasks (comparison, multiplication, or subtraction) but not in simple digit naming relative to the letter-naming control. Second, although the circuit was already engaged bilaterally during the number comparison task relative to control, the four numerical tasks could be ordered hierarchically in the order Naming < Comparison < Multiplication < Subtraction, so each higher-level task added a specific activation to the immediately lower task. Relative to digit naming, comparison only activated the depth of the right postcentral sulcus. Relative to comparison, multiplication caused a strong additional left intraparietal activation. Finally relative to multiplication, subtraction yielded greater right postcentral and bilateral prefrontal activation.

\section{A Parieto-Fronto-Cingular Network for Number Processing}

The network of areas active during number processing included parietal, frontal, and anterior cingulate components. In the parietal lobe, activation was concentrated along the banks of the intraparietal sulcus as well in the depth of the postcentral gyrus. In the frontal lobe, the active areas were distributed in the inferior (BA 44/45), dorsolateral (BA 46/9), and superior (BA 6/8) frontal gyri as well as the SMA and premotor cortex. Anatomically, these areas constitute a well-described network that is active in different cognitive tasks involving working memory and visuospatial attention (Corbetta, Miezin, Schulman, \& Petersen, 1993; Goldman-Rakic, 1984; Nobre et al., 1997). On the basis of anatomical tracing, lesion, and single-cell recording studies, Goldman-Rakic (1988) has proposed that different cognitive functions may be controlled within parallel distributed neural systems linking posterior parietal, prefrontal, and anterior cingulate cortices and related subcortical structures. Our results suggest that in humans, the internal manipulation of numbers is realized in such a circuit in close anatomical connection with the dorsal parietal pathway.

Part of the activations we observed, especially in the prefrontal and anterior cingulate cortex, are undoubtedly related to nonnumerical factors such as working memory and executive attention. Our numerical tasks were initially designed to require minimal contributions from working memory and strategical processes. On 
Table 3. Coordinates and Z Scores of Significant Activation Peaks When Numerical Tasks Were Contrasted

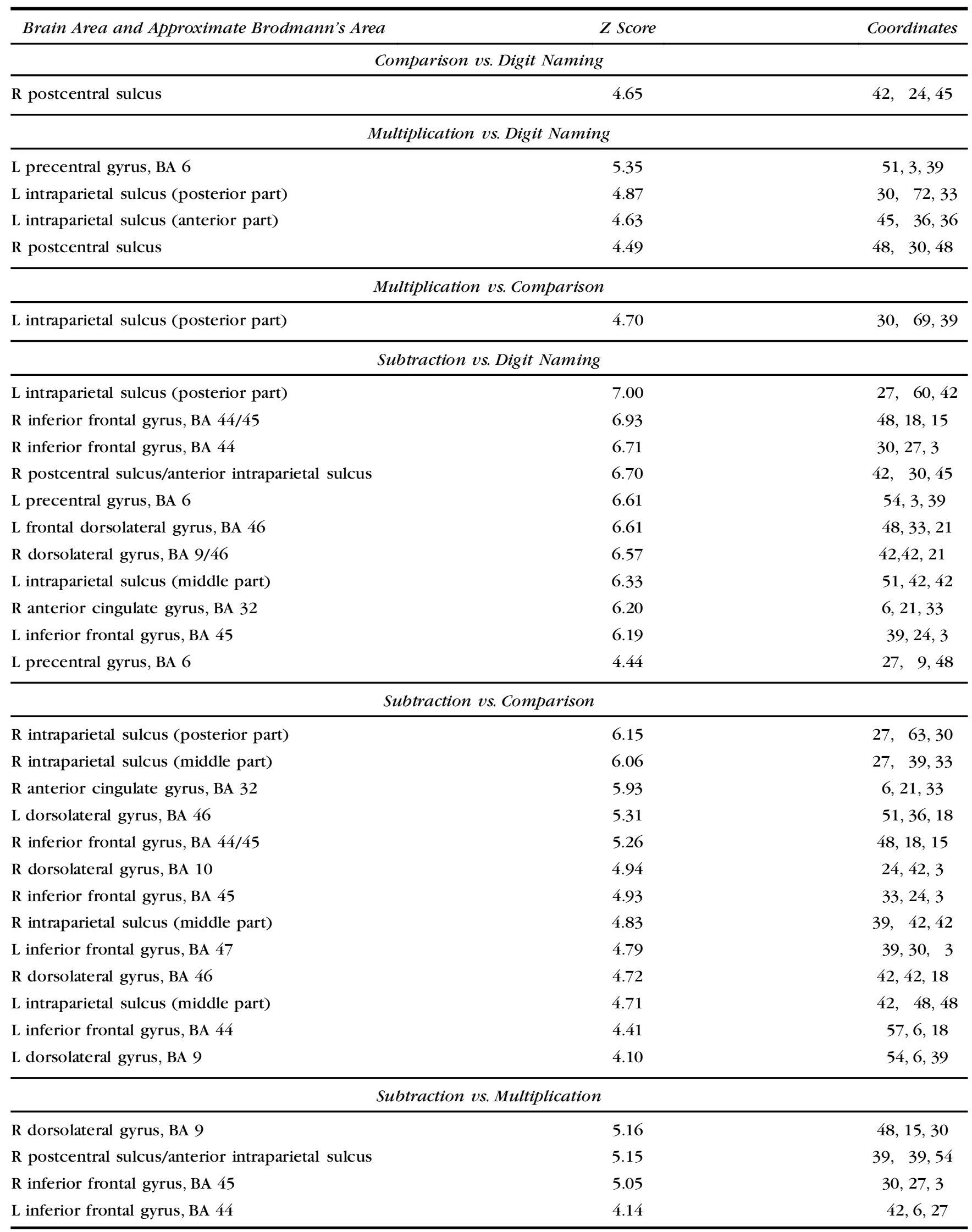


each trial, only a single digit was presented and a single internal operation was required. Yet in retrospect, there are several ways in which working memory might have been involved. First, the target digits were flashed for only $200 \mathrm{msec}$, after which they had to be kept in mind. Second, subjects were asked to keep in mind the second operand of each operation ( 3 for multiplication, 5 for comparison, and 11 for subtraction). Third, subjects reported a posteriori that the pace of the task implied that, for the most difficult multiplication and subtraction trials, on some trials they had not fully completed processing before the next target appeared; therefore they occasionally had to monitor two items in memory. Fourth, subjects also reported that on multiplication and subtraction trials, they often did not retrieve the result of, say, 118 from memory. Rather, they claimed to resort to simple strategies such as knowledge of sums totaling 10 (e.g., $11=10+1=(8+2)+1$, hence 11

$8=2+1=3$ ). Psychological research has indicated that even simple problems may require a strategical sequence of steps and hence the storage of intermediate results (LeFevre et al., 1996). Thus, working memory requirements may explain our observation of a strong activation in prefrontal cortex during simple calculation and also explain why this activation became more intense as the task increased in difficulty from digit naming to comparison, multiplication, and subtraction.

It seems unlikely, however, that working memory and attentional factors entirely explain the parietal lobe results. First, although the amount of activation was generally correlated with task difficulty as measured by reaction time and error rate, a single task-difficulty factor cannot explain the specific, nonlinear manner in which the left and right parietal activations emerged (right parietal activation in the comparison task, then left in the multiplication task; see Figure 4). Second, it is hard to see how our results could have been contaminated by an artifactual activation of the visuospatial attentional system. Our stimuli consisted of a single target digit (or a single letter in the control task) appearing at the center of the screen for $200 \mathrm{msec}$. Hence, there was no necessity for overt or covert spatial movement of gaze or attention. Furthermore, even if attention was required, for instance in the temporal domain to focus on the precise moment of appearance of the stimuli, there should be no difference with the control task of digit naming in that respect.

We envisage two alternative explanations for the strong parietal involvement in number processing. First, it may reflect the activation of a number-processing area anatomically close to but separate from the cerebral areas for visuospatial attention. Highly selective deficits for numbers can occur following an inferior parietal lesion of the dominant hemisphere (Dehaene \& Cohen, 1997; Warrington, 1982). Although parietal acalculia is frequently associated with agraphia, finger agnosia, and left-right confusion in a tetrad of symptoms called
Gerstmann's syndrome (Gerstmann, 1940), these deficits are dissociable (Benton, 1992), suggesting that knowledge of numbers may occupy its own specific cortical territory. Indeed, Dehaene and Cohen (1997) have suggested that acalculia in Gerstmann's syndrome is best described as a category-specific deficit for numbers, similar to the specific loss of knowledge that can occur for other categories of words such as animals, body parts, tools, or fruits and vegetables (Warrington \& McCarthy, 1987; Warrington \& Shallice, 1984). Patient MAR (Dehaene \& Cohen, 1997) could still read and write Arabic numerals but failed in tasks tapping elementary knowledge of numerical quantities such as computing 31 or deciding which number falls between 2 and 4 (although he could decide which letter falls between $B$ and $D$ or which month falls between February and April). Such evidence, together with data showing that infants and animals possess elementary numerical abilities and that early brain damage can result in a selective inability for arithmetic, has been taken to suggest that "number sense" is a biologically determined ability of the human, with a long evolutionary history and a specific cerebral substrate (Dehaene, 1997). According to this working hypothesis, the intraparietal activation might reflect the cerebral localization of a category-specific internal representation of numbers.

An alternative explanation is that the internal manipulation of numbers draws on visuospatial resources that are also recruited for genuinely spatial tasks. Experiments with normal subjects have revealed an intimate link between numbers and space. Whenever subjects process numbers, they respond faster on the right-hand side for larger numbers and on the left-hand side for smaller numbers, thus revealing an automatic spatialnumerical association, or SNARC, effect (Dehaene, Bossini, \& Giraux, 1993). Numbers seem to be represented internally in a spatially extended way, and the metaphor of a number line (Restle, 1970) has been proposed for the internal representation of numerical quantities (Dehaene, 1992; Gallistel \& Gelman, 1992). Indeed, a small fraction of normal subjects have the subjective experience of seeing a number line extended in two- or threedimensional space, often with rich details and colors (Galton, 1880; Seron, Pesenti, Noël, Deloche, \& Cornet, 1992). Spalding and Zangwill (1950) reported the case of a patient who claimed to have suddenly lost such a visual image of numbers and who experienced difficulties in calculating and in orienting in space following a lesion in the left parieto-occipital area. Restle (1970) suggested that subjects calculate by mentally moving along an oriented number line, for instance shifting attention one step to the left of 3 to compute $3 \quad 1$. The use of such spatial strategies for mental arithmetic might explain the activation of areas traditionally attributed to visuospatial attention during internal number processing tasks with no overt or covert attention-orienting components. 


\section{Dissociations between Numerical Operations}

In this section, we confront the results to our initial theoretical predictions about the dissociations between naming, comparing, multiplying, and subtracting numbers.

\section{An Asemantic Route for Number Naming?}

A first prediction was that the naming task would fail to strongly activate parietal areas associated with the semantic processing of numbers because a direct asemantic transcoding route is available for digit naming. This prediction was largely validated. Contrasting digit naming with letter naming revealed no activation of the parietal lobe at a conventional level of significance. ${ }^{2}$ The only activations were located in the right inferior frontal and right mesial frontal gyri. This suggests a greater right frontal contribution to number production than to letter production, a finding that may be related to the occasional dissociation of number production from the production of other words in either the spoken (Cohen, Verstichel, \& Dehaene, 1998) or the written modality (Anderson, Damasio, \& Damasio, 1990).

\section{Number Comparison and the Right Parietal Lobe}

A second prediction, derived from the triple-code model of number processing, was that number comparison should activate the left and right inferior parietal lobules, which are hypothesized to support a semantic representation of numerical quantities. Based on evidence from split-brain patients and those with major left-hemisphere lesions, we predicted that the right parietal lobule would play an important role in number comparison. The results confirmed this prediction. Both parietal lobes were activated, with a slight predominance for the right hemisphere. The right postcentral sulcus, in particular, was strongly solicited and was the only region to be activated during comparison relative to digit naming. This right-hemispheric predominance for number comparison fits well with the results of a recent event-related potential (ERP) study (Dehaene, 1996). In a task identical to the present one (comparison with a fixed standard of 5), a right-lateralized parieto-occipito-temporal ERP component was shown to be significantly affected by the distance between the target numbers and 5 but not by the notation used for the numbers (spelled-out numerals or Arabic digits) or by the hand used for responding. Dipole modeling showed that this distance electrical effect, which indexes the critical step of quantity comparison in this task, was consistent with a bilateral generator located deep in the left and right inferior parietal areas, with a stronger activity in the right hemisphere.

More surprising is the activation of the frontal cortex, anterior cingulate, and right putamen during number comparison relative to letter naming. These areas were not predicted by available models of number processing. As noted above, they might be related to processes not specific to numbers but inherent to the comparison task, such as working memory for the reference number, response decision, execution, or inhibition of digit naming and calculation. In an ERP study of number comparison, Dehaene et al. (1996) have reported an activation of the anterior cingulate cortex related to error monitoring and correction, which may have contributed to the present task.

\section{Multiplication versus Subtraction}

Our third prediction was that multiplication and subtraction, although superficially similar, would yield different activation patterns, with a greater bilateral inferior parietal involvement during subtraction and a strict left-hemispheric lateralization, with activation of perisylvian language areas, during multiplication. This prediction was only partially supported by the data. Certainly, subtraction entailed a considerable bilateral activation of the intraparietal sulcus, particularly relative to number comparison (Figure 4). Furthermore, activation was highly left-lateralized during multiplication, being confined to the left intraparietal area during multiplication relative to comparison. However, the direct contrast between multiplication and subtraction revealing only a few differences: Several prefrontal areas and the right postcentral region were significantly more active during subtraction, whereas no area was significantly more active during multiplication. The predicted activation of language areas during multiplication was remarkably absent. ${ }^{3}$ One possibility is that these areas were already present in all control conditions (because subjects always had to name the result) and were therefore canceled out in all contrasts. Indeed, exact resolution of addition problems strongly activated the left inferior frontal region and the left angular gyrus, among other areas, in a recent study in which the control task involved the presentation of letters but did not require naming (Dehaene et al., 1999).

The association of multiplication with the left intraparietal area, although not predicted by our theoretical framework, is clearly compatible with previous findings. With positron emission tomography (PET), Dehaene et al. (1996) reported bilateral inferior parietal activation, with a left lateralization, during a multiplication task. With ERPs, Kiefer and Dehaene (1997) also found left lateralized inferior parietal activity during both simple and complex multiplication facts, with a tendency for a later bilateral activation for complex multiplication facts only. These observations must be reconciled with the observation that parietal lesions that affect number comprehension may leave multiplication retrieval partially intact (Dehaene \& Cohen, 1997; Delazer \& Benke, 1997). 
A plausible explanation is that the robust parietal activation during multiplication reflects quantity-based processes that are useful to normal subjects but are not strictly needed for the task. When solving even simple multiplication problems, normal subjects often use a combination of direct retrieval and quantity-based strategies (Campbell, 1994; LeFevre et al., 1996). For instance, the order of the operands may be reversed $(3 \times 8=8 \times$ $3=24$ ) or the problem may be decomposed into simpler facts $(3 \times 5=5+5+5=15)$. Such "semantic elaboration" strategies require an understanding of the quantities involved in the original problem, which would be expected to result in inferior parietal activation (Dehaene \& Cohen, 1995). Given the replicability of this activation, the triple-code model should acknowledge that the semantic representation of numerical quantities makes an important, although perhaps optional, contribution to the retrieval of arithmetic facts.

\section{CONCLUSION}

The present results establish both the existence of a parieto-fronto-cingulate network active during various mental arithmetic tasks and its variable involvement as a function of task demands. The left and right parietal regions, although they both contribute to mental arithmetic, may not be functionally equivalent. At present, we only have little cues about what these functions may be. It is noteworthy, however, that a task calling only for the internal manipulation of numerical quantity, number comparison, was found to rely more on the right parietal lobule, whereas a task presumably requiring access to verbal memory was more strongly associated with the left parietal lobule. Our working hypothesis, which we would like to tentatively propose in this conclusion, is that although both parietal areas are involved in manipulating quantity information, only the left parietal region provides the interconnection of the quantity representation with the linguistic code. Indeed, this is a direct consequence of the triple-code model, in which the left inferior parietal region provides the only direct connection between the left verbal system and the right parietal quantity system (Figure 1). During multiplication, the left parietal region would be strongly activated because subjects use the quantity representation to monitor the plausibility of the results they have obtained through verbal computations, as suggested above. During comparison, the right parietal region would suffice, because comparison involves accessing the quantity system from the Arabic notation but does not require any translation between the verbal and quantity formats. During subtraction, finally, both the left and the right parietal lobules would be active because subtraction requires both internal quantity manipulations and naming of the resulting quantity. The pivotal role of the left parietal region would also explain why left, but not right, inferior parietal lesions yield strong impairments of calculation.

\section{METHOD}

\section{Subjects}

Eight right-handed subjects (four women and four men) aged between 20 and 30 years participated in the imaging study. All were drug free, had no neurological or psychiatric history, and had normal anatomical magnetic resonance images. All gave their written informed consent. The experiment was approved by the Ethical Committee of the Hôpital de Bicêtre, Paris.

\section{Stimuli}

In the imager, visual stimuli were projected on a translucent screen placed at the subject's head. Stimuli were displayed using an active-matrix video projector controlled by a PC computer running the EXPE5 software for millisecond timing (Pallier, Dupoux, \& Jeannin, 1997). Subjects wore a head-mounted mirror that allowed them to see the stimuli in their normal upright position. The same stimuli were used for the four numerical tasks (naming, comparison, multiplication, and subtraction): Random digits between 1 and 9, excluding digit 5, were flashed for $200 \mathrm{msec}$, at a rate of one every $2 \mathrm{sec}$. For the control task, random letters between $\mathrm{A}$ and I, excluding letter $\mathrm{E}$, were flashed using the same parameters of duration and rate. Letters and digits were presented in alternating blocks of 18 trials (36 sec) each.

\section{Tasks}

To prevent head movements, subjects were told to perform all the tasks mentally, without overt vocalization. During letter blocks, they named the letters mentally. During the digit blocks, they performed one of the following four numerical tasks. In the naming task, subjects had to name the target digit. In the comparison task, subjects were instructed to compare the target digit to the standard number 5, mentally saying "larger" or "smaller." In the multiplication task, subjects had to multiply the target digit by 3 and then to name the result mentally. In the subtraction task, subjects had to subtract the target digit from 11 and to name the result mentally. For each task, the paradigm consisted in three experimental blocks alternating with three control blocks. Thus, each experiment included four runs of $336 \mathrm{sec}$, (i.e., one run for each experimental task).

\section{Data Acquisition}

All experiments were performed on a 3-T whole-body system (Bruker, Germany) equipped with a quadrature 
birdcage radio frequency (RF) coil and a head-gradient coil insert designed for echoplanar imaging. Foam padding was used to limit head motion within the coil. Functional images were obtained with a T2*-weighted gradient echo, echo planar imaging sequence $(T R=6000$ msec, $\mathrm{TE}=40 \mathrm{msec}, \mathrm{FOV}=220 \times 220 \mathrm{~mm}^{2}$, matrix $=$ $64 \times 64$ ), using blood oxygen level-dependent contrast. Eighteen 5-mm-thick axial slices covering most of the brain were acquired every 6 sec. Thirty-nine images, each consisting of 18 slices, were collected consecutively for each task. The first three images were not included in the analysis. Functional images were reconstructed and analyzed off-line. High-resolution images (3-D gradientecho inversion-recovery sequence, $\mathrm{TI}=700 \mathrm{msec}, \mathrm{TR}=$ $1600 \mathrm{msec}, \mathrm{FOV}=192 \times 256 \times 256 \mathrm{~mm}^{3}$, matrix $=256 \times$ $128 \times 256$, slice thickness $=1 \mathrm{~mm}$ along head-foot axis) were also acquired for anatomical localization.

\section{Data Analysis}

All subsequent data analyses were performed with Statistical Parametric Mapping, version 96 (SPM96). To correct for motion, the scans from each subject were realigned using the last image as a reference (the image whose acquisition time is nearest to that of anatomical images). For each subject, anatomical images were transformed stereotactically to Talairach coordinates using the standard template of the Montreal Neurological Institute. The functional scans were then normalized using the same transformation. Functional images were smoothed with a Gaussian spatial filter of $5 \mathrm{~mm}$. The resulting images had cubic voxels of $3 \times 3 \times 3 \mathrm{~mm}^{3}$, and the final image resolution was $7.3 \times 7.3 \times 7.2 \mathrm{~mm}^{3}$. The anatomical images had cubic voxels of $2 \times 2 \times 2 \mathrm{~mm}^{3}$.

Each block of activation was modeled by two temporal basis functions, the first one for the early component of the activation and the second one for the later component. We used a high-pass filter set at $120 \mathrm{sec}$, roughly twice the period of the paradigm. Individual data were analyzed using a randomized block design with global brain activity as a covariate of noninterest. After statistical analysis and for each subject, the activation maps were superimposed on individual anatomical images for localization purposes, with the support of their Talairach coordinates.

For the group analysis, we used a voxelwise significance threshold of 0.001 , corrected to $p<0.05$ for multiple comparisons by the standard procedure of SPM96. With the particular statistical parameters of our images, this corresponded to reporting only clusters with more than 16 neighboring voxels, each active at $p<0.001$. To identify active areas, we first examined a contrast comparing the main effect of the four numerical tasks relative to the letter-naming control. Then we examined the four contrasts, digit naming $>$ control, comparison $>$ control, multiplication $>$ control, and subtraction $>$ control, to identify the areas involved in each numerical task. Finally, we also analyzed the 12 contrasts corresponding to all possible comparisons between two numerical tasks. Because each numerical task was acquired in a distinct block, these between-task contrasts were framed as interaction terms in SPM96. For instance, to compare multiplication with subtraction, we used the following interaction term: (multiplication its letter-naming control) (subtraction its letter-naming control). We masked these contrasts with the original contrast of the appropriate task relative to control. For instance, the above contrast for multiplication $>$ subtraction was masked by the original contrast multiplication $>$ letter-naming control (at $p<0.001$ ). This ensured that we looked only at areas that showed significant differences across tasks and were active relative to control. Significant differences that were due to a greater deactivation in one task relative to the other, whose interpretation is difficult, were canceled out by this procedure.

The same statistical analysis was applied separately to each individual subject. Because of the smaller number of degrees of freedom, a voxelwise significance threshold of 0.001 corrected to $p<0.1$ was then used. Details of the individual analyses are available from the authors. Here we only report, for each significant effect in the group analysis, the number of subjects who showed that effect in the same anatomical area in the individual analysis.

\section{Behavioral Control Study}

Eight additional subjects were run in a behavioral control study. The same stimuli were presented on a standard PC monitor in five blocks of 56 trials each, corresponding to the five tasks (letter naming, digit naming, comparison, multiplication, and subtraction). Subjects spoke their responses aloud in a voice-activated relay. Vocal reaction times were measured to the closest millisecond, and responses were recorded for subsequent scoring of errors. Each trial consisted of an initial 2000-msec blank screen. The stimulus was then flashed for $200 \mathrm{msec}$. The subject's vocal response triggered the next trial. The five tasks were presented in random order.

\section{Acknowledgments}

This work was supported by INSERM, the Groupement d'Intérêt Scientifique (GIS) "Sciences de la Cognition," and the Fondation pour la Recherche Médicale (FRM). We thank E. Giacomini, D. Le Bihan, G. Le Clec'h, S. Lehéricy and J. B. Poline for their technical and statistical help.

Reprint requests should be sent to Stanislas Dehaene, INSERM U334, Service Hospitalier Frédéric Joliot, CEA/DSV, 4 place du Général Leclerc, 91401 Orsay Cedex, France, or via e-mail: dehaene@shfj.cea.fr. 


\section{Notes}

1. This patient, MAR, was unusual in that he showed Gerstmann's syndrome following a right inferior parietal lesion. The patient was left-handed, however, and might have had an unusual lateralization pattern. More recently, the dissociation between severely impaired subtraction and relatively more preserved multiplication was replicated in several cases of acalculia and Gerstmann's syndrome stemming from a classical left inferior parietal lesion (Delazer \& Benke, 1997; L. Cohen and S. Dehaene, 1997, unpublished observations).

2. In various chronometric tasks, including naming, the mere presentation of a digit on a screen suffices to induce a quantity-based interference in response times (Brysbaert, 1995; Dehaene \& Akhavein, 1995; Dehaene et al., 1998; LeFevre, Bisanz, \& Mrkonjic, 1988). Thus, one might have expected an automatic activation of the parietal quantity system during naming, even if it was not strictly required for the task. We therefore reexamined the presence of subthreshold parietal activation during the naming task at a lower level of significance. We first used the data from the subtraction condition to identify seven active voxels related to number processing in the inferior parietal lobules (at the conventional level of significance: $p<0.001$, corrected for multiple comparisons to $p<0.05$ ). We then asked whether these voxels showed a significance difference in the contrast of naming versus control, now at the lower significance of $p<0.05$. This was indeed the case. All seven parietal activation peaks listed in Table 1 showed a small increase in activation during digit naming as compared to letter naming, significant at $p<0.05$. In fact, two major clusters of 104 and 71 voxels, respectively, were activated at $p<0.05$ in the left and right intraparietal/postcentral area during digit naming compared to letter naming.

3. The left basal ganglia have been tentatively implicated in the retrieval of rote multiplication facts (Dehaene \& Cohen, 1995). Here, we did not find left subcortical involvement in multiplication with standard statistical thresholds. Because those thresholds required at least 16 contiguous voxels (432 $\mathrm{mm}^{3}$ ), each with $p<0.001$, for a cluster of active voxels to be considered significant, we also reexamined subcortical activity without imposing a minimum cluster size, but with a stringent voxelwise threshold of $p<0.0001$. Although no activation was found in subtraction versus letter naming, we did find a single subcortical activation in the head of the left caudate nucleus ( $18,8,22 ; Z=3.90 ; 5$ voxels) in multiplication versus letter naming. This activation, although still present in multiplication versus digit naming, was not present when multiplication was contrasted with either comparison or subtraction, even at $p<$ 0.05 . Thus, the evidence for a specific role of the left basal ganglia in multiplication remained weak at best.

\section{REFERENCES}

Anderson, S. W., Damasio, A. R., \& Damasio, H. (1990). Troubled letters but not numbers: Domain specific cognitive impairments following focal damage in frontal cortex. Brain, 113, 749-766.

Benton, A. L. (1992). Gerstmann's syndrome. Archives of Neurology, 49, 445-447.

Brysbaert, M. (1995). Arabic number reading: On the nature of the numerical scale and the origin of phonological recoding. Journal of Experimental Psychology: General, 124, 434-452.

Campbell, J. I. D. (1994). Architectures for numerical cognition. Cognition, 53, 1-44.

Cipolotti, L., \& Butterworth, B. (1995). Toward a multiroute model of number processing: Impaired number transcod- ing with preserved calculation skills. Journal of Experimental Psychology: General, 124, 375-390.

Cohen, L., \& Dehaene, S. (1995). Number processing in pure alexia: The effect of hemispheric asymmetries and task demands. NeuroCase, 1, 121-137.

Cohen, L., \& Dehaene, S. (1996). Cerebral networks for number processing: Evidence from a case of posterior callosal lesion. NeuroCase, 2, 155-174.

Cohen, L., Verstichel, P., \& Dehaene, S. (1998). Neologistic jargon sparing numbers: A category-specific phonological impairment. Cognitive Neuropsychology, 14, 1029-1061.

Corbetta, M., Miezin, F. M., Schulman, G. L., \& Petersen, S. E. (1993). A PET study of visuospatial attention. Journal of Neuroscience, 13, 1202-1226.

Dagenbach, D., \& McCloskey, M. (1992). The organization of arithmetic facts in memory: Evidence from a brain-damaged patient. Brain and Cognition, 20, 345-366.

Dehaene, S. (1992). Varieties of numerical abilities. Cognition, 44, 1-42.

Dehaene, S. (1996). The organization of brain activations in number comparison: Event-related potentials and the additive-factors methods. Journal of Cognitive Neuroscience, 8, 47-68.

Dehaene, S. (1997). The number sense. New York: Oxford University Press.

Dehaene, S., \& Akhavein, R. (1995). Attention, automaticity and levels of representation in number processing. Journal of Experimental Psychology: Learning, Memory, and Cognition, 21, 314-326.

Dehaene, S., Bossini, S., \& Giraux, P. (1993). The mental representation of parity and numerical magnitude. Journal of Experimental Psychology: General, 122, 371-396.

Dehaene, S., \& Cohen, L. (1991). Two mental calculation systems: A case study of severe acalculia with preserved approximation. Neuropsychologia, 29, 1045-1074.

Dehaene, S., \& Cohen, L. (1995). Towards an anatomical and functional model of number processing. Matbematical Cognition, 1, 83-120.

Dehaene, S., \& Cohen, L. (1997). Cerebral pathways for calculation: Double dissociation between rote verbal and quantitative knowledge of arithmetic. Cortex, 33, 219-250.

Dehaene, S., Naccache, L., Le Clec'h, G., Koechlin, E., Mueller, M., Dehaene-Lambertz, G., van de Moortele, P. F., \& Le Bihan, D. (1998). Imaging unconscious semantic priming. $\mathrm{Na}$ ture, 395, 597-600.

Dehaene, S., Spelke, E., Stanescu, R., Pinel, P., \& Tsivkin, S. (1999). Sources of mathematical thinking: Behavioral and brain-imaging evidence. Science, 284, 970-974.

Dehaene, S., Tzourio, N., Frak, V., Raynaud, L., Cohen, L., Mehler, J., \& Mazoyer, B. (1996). Cerebral activations during number multiplication and comparison: A PET study. Neuropsychologia, 34, 1097-1106.

Delazer, M., \& Benke, T. (1997). Arithmetic facts without meaning. Cortex, 33, 697-710.

Gallistel, C. R., \& Gelman, R. (1992). Preverbal and verbal counting and computation. Cognition, 44, 43-74.

Galton, F. (1880). Visualized numerals. Nature, 21, 252-256.

Gazzaniga, M. S., \& Hillyard, S. A. (1971). Language and speech capacity of the right hemisphere. Neuropsychologia, 9, 273-280.

Gazzaniga, M. S., \& Smylie, C. E. (1984). Dissociation of language and cognition: A psychological profile of two disconnected right hemispheres. Brain, 107, 145-153.

Gerstmann, J. (1940). Syndrome of finger agnosia disorientation for right and left agraphia and acalculia. Archives of Neurology and Psychiatry, 44, 398-408.

Goldman-Rakic, P. S. (1984). Modular organization of prefrontal cortex. Trends in Neuroscience, 7, 419-424. 
Goldman-Rakic, P. S. (1988). Topography of cognition: Parallel distributed networks in primate association cortex. Annual Review of Neuroscience, 11, 137-156.

Grafman, J., Kampen, D., Rosenberg, J., Salazar, A., \& Boller, F. (1989). Calculation abilities in a patient with a virtual left hemispherectomy. Behavioral Neurology, 2, 183-194.

Kiefer, M., \& Dehaene, S. (1997). The time course of parietal activation in single-digit multiplication: Evidence from event-related potentials. Mathematical Cognition, 3, 1-30.

Lampl, Y., Eshel, Y., Gilad, R., \& Sarova-Pinhas, I. (1994). Selective acalculia with sparing of the subtraction process in a patient with left parietotemporal hemorrhage. Neurology, 44, 1759-1761.

Langdon, D. W., \& Warrington, E. K. (1997). The abstraction of numerical relations: A role for the right hemisphere in arithmetic? Journal of the International Neuropsychological Society, 3, 260-268.

LeFevre, J. A., Bisanz, J., Daley, K. E., Buffone, L., Greenbaum, S. L., \& Sadesky, G. S. (1996). Multiple routes to solution of single-digit multiplication problems. Journal of Experimental Psychology: General, 125, 284-306.

LeFevre, J., Bisanz, J., \& Mrkonjic, L. (1988). Cognitive arithmetic: Evidence for obligatory activation of arithmetic facts. Memory \& Cognition, 16, 45-53.

McNeil, J. E., \& Warrington, E. K. (1994). A dissociation between addition and subtraction within written calculation. Neuropsychologia, 32, 717-728.

Nobre, A. C., Sebestyen, G. N., Gitelman, D. R., Mesulam, M. M., Frackowiak, R. S. J., \& Frith, C. D. (1997). Functional localization of the system for visuospatial attention using positron emission tomography. Brain, 120, 515-533.

Pallier, C., Dupoux, E., \& Jeannin, X. (1997). EXPE5: An expandable programming language for on-line psychological experiments. Behavior Research, Methods, Instruments and Computers, 29, 322-327.

Pesenti, M., Seron, X., \& van der Linden, M. (1994). Selective impairment as evidence for mental organization of arithmetical facts: $\mathrm{BB}$, a case of preserved subtraction? Cortex, 30, 661-671.

Restle, F. (1970). Speed of adding and comparing numbers. Journal of Experimental Psychology, 91, 191-205.

Roland, P. E., \& Friberg, L. (1985). Localization of cortical areas activated by thinking. Journal of Neurophysiology, 53, 1219-1243.

Rosselli, M., \& Ardila, A. (1989). Calculation deficits in patients with right and left hemisphere damage. Neuropsychologia, 27, 607-617.

Rueckert, L., Lange, N., Partiot, A., Appollonio, I., Litvar, I., Le Bihan, D., \& Grafman, J. (1996). Visualizing cortical activation during mental calculation with functional MRI. Neuroimage, 3, 97-103.

Seron, X., Pesenti, M., Noël, M. P., Deloche, G., \& Cornet, J.-A. (1992). Images of numbers or when 98 is upper left and 6 sky blue. Cognition, 44, 159-196.

Seymour, S. E., Reuter-Lorenz, P. A., \& Gazzaniga, M. S. (1994). The disconnection syndrome: Basic findings reaffirmed. Brain, 117, 105-115.

Spalding, J. M. K., \& Zangwill, O. L. (1950). Disturbance of number-form in a case of brain injury. Journal of Neurology, 13, 24-29.

Takayama, Y., Sugishita, M., Akiguchi, I., \& Kimura, J. (1994). Isolated acalculia due to left parietal lesion. Archives of Neurology, 51, 286-291.

Warrington, E. K. (1982). The fractionation of arithmetical skills: A single case study. Quarterly Journal of Experimental Psychology, 34A, 31-51.

Warrington, E. K., \& McCarthy, R. (1987). Categories of knowledge: Further fractionation and an attempted integration. Brain, 110, 1273-1296.

Warrington, E. K., \& Shallice, T. (1984). Category-specific semantic impairments. Brain, 107, 829-854. 